\title{
Terapia con metformina e rischio di acidosi lattica
}

\author{
Angela Dardano, Daniele Giuseppe \& Stefano Del Prato
}

\author{
Dipartimento di Medicina Clinica e Sperimentale, Sezione di Malattie del Metabolismo e \\ Diabetologia, \\ Università̀ di Pisa, Via Paradisa 2, 56124, Pisa, Italia
}

Oltre cinquanta anni fa, il farmacologo francese Jean Sterne pubblicava il primo studio clinico su una biguanide diventata pietra angolare nella storia della diabetologia: la metformina (Met).

Da allora, la Met rappresenta il farmaco di riferimento nella cura dei soggetti affetti da diabete mellito tipo 2 (T2D) in ogni fase della malattia e può essere utilizzata in monoterapia 0 in associazione ad altri ipoglicemizzanti, inclusa l'insulina (1). Inoltre, nei soggetti con pre-diabete, in aggiunta all'intervento sullo stile di vita, si è dimostrata efficace nel prevenire (o ritardare) la comparsa di T2D. Le comprovate evidenze di efficacia, il profilo di sicurezza clinica (rischio di ipoglicemia pressoché assente) e i dati di protezione cardiovascolare generati dallo "United Kingdom Prospective Diabetes Study" (UKPDS) hanno reso la Met il farmaco di prima linea nel trattamento del T2D. Studi recenti hanno anche suggerito una potenziale azione anti-cancerogena, anti-infiammatoria e anti-aging della Met, conferendo alla molecola le caratteristiche di pleiotropismo (2). Dal punto di vista farmacocinetico, la Met è assorbita nel tratto gastrointestinale superiore (duodeno e digiuno prossimale), si concentra negli enterociti e negli epatociti, circola non legata alle proteine plasmatiche e viene eliminata dal rene in forma immodificata (3). Gli effetti collaterali più comuni della Met sono quelli a carico del tratto gastrointestinale (in media circa il 10\% dei soggetti trattati), che di solito sono transitori, tempo e dose-dipendenti e più frequenti nei soggetti già affetti da patologie intestinali. Le formulazioni a lento rilascio, recentemente introdotte anche nel nostro Paese, sono associate a una tolleranza ancora maggiore e permettono la mono-somministrazione nella maggior parte dei casi. La preoccupazione principale associata alla terapia con Met è il rischio di acidosi lattica, condizione rara ( $\leq 10$ casi su 100.000 pazienti trattati per anno), ma potenzialmente "life-threatening" (rischio di mortalità 30$50 \%)$ (4). Per tale motivo, nel drug labeling, le raccomandazioni sull'uso della Met sono ancora molto restrittive, in particolare nell'insufficienza renale cronica, per il possibile accumulo del farmaco, anche se fonti autorevoli hanno di recente proposto la possibilità di impiegare la Met con cautela fino a valori di filtrato glomerulare stimato di $30 \mathrm{ml} / \mathrm{min} / 1,73 \mathrm{~m} 2$, purché siano attentamente considerati $i$ fattori di rischio di peggioramento della funzione renale $(5,6,7)$. Studi preclinici hanno dimostrato che l'acidosi lattica è correlata alla dose e alla concentrazione plasmatica di biguanide, sebbene la forma di acidosi associata a Met (MALA) sia circa 20 volte inferiore rispetto a quella osservata con la fenformina. II reale tasso di incidenza di MALA è, tuttavia, difficile da stimare sia per la presenza in letteratura di "case reports" sia per la difficoltà di dosare la concentrazione plasmatica di metformina, elemento essenziale della triade diagnostica, insieme a elevati livelli di acido lattico ( $>5 \mathrm{mmol} / \mathrm{l}$ ) e riduzione del $\mathrm{pH}$ arterioso (<7.35), in assenza di ipoperfusione. Quando la Met è implicata nei casi di acidosi, di solito le concentrazioni plasmatiche sono superiori a $5 \mathrm{\mu g} / \mathrm{ml}$. Bisogna, comunque, ricordare che tecniche non perfettamente eseguite del prelievo di sangue e della sua conservazione prima della misurazione dell'acido lattico possono dare risultati poco attendibili (8) e che generalmente la concentrazione di Met rimane inferiore a $2 \mu \mathrm{g} / \mathrm{ml}$, almeno nel range del dosaggio terapeutico $(500-3000 \mathrm{mg} / \mathrm{die})$. La meta-analisi della Cochrane Database non ha osservato un aumentato rischio di acidosi lattica in corso di terapia con Met rispetto ad altri trattamenti antiperglicemici, quando prescritta con cautela e in assenza di controindicazioni (9). II 
meccanismo d'azione della Met è complesso e non ancora ben definito. Al momento si ritiene che la Met agisca principalmente inibendo la gluconeogenesi epatica e si suppone che alteri i substrati del complesso I della catena respiratoria mitocondriale. Mediante l'inibizione della catena respiratoria mitocondriale, la glicolisi aerobia è bloccata e si ha un incremento della glicolisi anaerobia, con accumulo di acido lattico (10). Nella maggior parte dei casi, un aumento dei livelli di acido lattico in corso di terapia con Met è stato osservato in presenza di severa riduzione della funzione renale (ridotta clearance della Met), compromissione della funzione epatica (ridotto metabolismo dell'acido lattico) e/o aumentata produzione di lattato (sepsi, insufficienza cardiaca, ipoperfusione) (Figura). I soggetti affetti da T2D sembrano essere più a rischio di sviluppare acidosi per difetti del potenziale redox e sono, quindi, più vulnerabili allo sviluppo di acidosi in risposta a un evento secondario. In questo contesto, sebbene non rappresentino controindicazioni assolute, un'età avanzata, condizioni a rischio di disidratazione, alcoolismo, interventi chirurgici e l'impiego di mezzo di contrasto iodato o di farmaci interferenti con l'emodinamica renale sono condizioni nelle quali un uso prudente, se non la sospensione del trattamento con Met, dovrebbe essere raccomandato. In studi preclinici la somministrazione di Met a livello dell'ileo non comportava incrementi dei livelli di acido lattico (10). In linea con queste osservazioni, studi clinici, in cui è stata impiegata la formulazione di Met a rilascio ritardato, hanno evidenziato un'azione del farmaco prevalente a livello ileale, con modesto aumento delle concentrazioni circolanti del farmaco, suggerendo una possibile riduzione degli effetti indesiderati tra cui l'aumento di acido lattico (11). Questo potrebbe fornire nuove e interessanti prospettive sull'impiego di Met, in particolare nelle popolazioni a più alto rischio di MALA.

In conclusione, l'uso della metformina da oltre mezzo secolo rende il farmaco sicuro e di prima scelta nel trattamento del T2D, anche per i suoi effetti pleiotropici. L'acidosi lattica è un evento raro, seppur grave, ma largamente evitabile. I soggetti con MALA hanno, infatti, spesso co-morbilità o fattori di rischio che contribuiscono allo sviluppo dell'acidosi. Nuove formulazioni di Met, con meccanismo d'azione "intestinale", possono rappresentare una scelta strategica per prevenire il rischio di acidosi lattica in soggetti predisposti, ridurre la mortalità e preservare l'omeostasi fisiologica.

\section{Riferimenti Bibliografici}

1. Inzucchi SE, Bergenstal RM, Buse JB, Diamant M, Ferrannini E, Nauck M, Peters AL, Tsapas A, Wender R, Matthews DR; American Diabetes Association (ADA); European Association for the Study of Diabetes (EASD). Management of hyperglycemia in type 2 diabetes: a patient-centered approach: position statement of the American Diabetes Association (ADA) and the European Association for the Study of Diabetes (EASD). Diabetes Care. 2012;35:1364-79

2. Fujita Y, Inagaki N. Metformin: New Preparations and Nonglycemic Benefits. Curr Diab Rep. 2017 Jan;17(1):5.

3. Bailey CJ, Turner RC. Metformin. N Engl J Med 1996 Feb 29;334(9):574-9.

4. Misbin RI, Green L, Stadel BV, Gueriguian JL, Gubbi A, Fleming GA. Lactic acidosis in patients with diabetes treated with metformin. N Engl J Med. 1998 Jan 22;338(4):265-6

5. Glucophage (metformin hydrochloride) and Glucophage XR (extended release) prescribing information. Bristol, NJ: Bristol Myers Squibb; 2009

6. Inzucchi SE, Lipska KJ, Mayo H, Bailey CJ, McGuire DK. Metformin in patients with type 2 diabetes and kidney disease: a systematic review. JAMA. 2014 Dec 24-31;312(24):2668-75.

7. Standard Italiani per cura del diabete mellito AMD - SID. http://www.standarditaliani.it/

8. Burnett RW, Covington AK, Fogh-Andersen N, Külpmann WR, Maas AH, Müller-Plathe O, SiggaardAndersen O, Van Kessel AL, Wimberley PD, Zijlstra WG. International Federation of Clinical Chemistry (IFCC). Scientific Division. Committee on pH, Blood Gases and Electrolytes. Approved 
IFCC recommendations on whole blood sampling, transport and storage for simultaneous determination of $\mathrm{pH}$, blood gases and electrolytes. Eur J Clin Chem Clin Biochem. 1995 Apr;33(4):247-53

9. Salpeter SR, Greyber E, Pasternak GA, Salpeter EE. Risk of fatal and nonfatal lactic acidosis with metformin use in type 2 diabetes mellitus: systematic review and meta-analysis. Cochrane Database Syst Rev. 2010 Apr 14;(4):CD002967.

10. DeFronzo R, Fleming GA, Chen K, Bicsak TA. Metformin-associated lactic acidosis: Current perspectives on causes and risk. Metabolism. 2016 Feb;65(2):20-9

11. Buse JB, DeFronzo RA, Rosenstock J, Kim T, Burns C, Skare S, Baron A, Fineman M. The Primary Glucose-Lowering Effect of Metformin Resides in the Gut, Not the Circulation: Results From Shortterm Pharmacokinetic and 12-Week Dose-Ranging Studies. Diabetes Care. 2016 Feb;39(2):198-205 